Revista Calidad en la Educación Superior

Programa de Autoevaluación Académica

Universidad Estatal a Distancia

ISSN 1659-4703

Costa Rica

revistacalidad@uned.ac.cr

\title{
LA EVALUACIÓN CUALITATIVA Y LA EVALUACIÓN FORMATIVA COMO PARTE DE LA ESTRATEGIA DIDÁCTICA EN CURSOS DE CAPACITACIÓN.

\author{
QUALITATIVE EVALUATION AND FORMATIVE EVALUATION AS PART OF \\ THE TEACHING STRATEGY IN TRAINING.
}

\begin{abstract}
Olga Amador Castro, oamador@uned.ac.cr ${ }^{1}$ Julia Pérez Chaverri, jperez@uned.ac.cr ${ }^{2}$ Centro de Capacitación en Educación a Distancia Universidad Estatal a Distancia, Costa Rica Aprender para evaluar y Evaluar para Aprender I Simposio sobre Evaluación de los Aprendizajes
\end{abstract}

\author{
Volumen 5, número 2 \\ Noviembre, 2014 \\ pp. $239-262$
}

Recibido: agosto, 2014

Aprobado: octubre, 2014

\footnotetext{
1 Funcionaria del Centro de Capacitación en Educación a Distancia, Universidad Estatal a Distancia, Costa Rica.

2 Funcionaria del Centro de Capacitación en Educación a Distancia, Universidad Estatal a Distancia, Costa Rica.
} 


\title{
Resumen
}

Desde hace un año se empezaron a implementar acciones dentro de los cursos que ofrece el Centro de Capacitación en Educación a Distancia (CECED), con el fin de potenciar las evaluaciones cualitativa y formativa, tal como se enmarca en el modelo pedagógico de la Universidad Estatal a Distancia (UNED). En ese sentido, esta ponencia pretende exponer diversas percepciones de algunos/as funcionarios/as académicos/as que participaron en cursos de capacitación en los que se implementaron dichas prácticas evaluativas, las cuales también pueden ser desarrolladas en las diferentes ofertas académicas de nuestra universidad.

Estas percepciones son el resultado de los conocimientos y las experiencias de las personas participantes en la última oferta de los cursos Elaboración de Artículos Académicos del III cuatrimestre de 2013, y Elaboración de Ensayos Académicos y Organización y Diseño de Cursos en Línea del I cuatrimestre de 2014, procesos de capacitación que se desarrollaron en la plataforma virtual Moodle. Estos cursos incorporan el tema de la evaluación cualitativa y la formativa tanto en lo teórico como en lo práctico, lo cual se evidencia en la propuesta evaluativa del curso y en el papel que asume la persona facilitadora de este (capacitador).

Palabras clave: evaluación cualitativa, evaluación formativa, evaluación auténtica, cursos virtuales, educación a distancia.

\begin{abstract}
Years ago the Training Center for Distance Education (CECED, for its acronym in Spanish) started to implement actions within the courses that offered in order to enhance the qualitative and formative evaluation, as part of the pedagogical model of the Universidad Estatal a Distancia (UNED). In that sense, this paper tries to present different perceptions of some academics who participated in training courses where such evaluation practices were applied, and which can also be developed in the different academic offers that our University implements. These perceptions are the result of the knowledge and experiences of the participants in the latest offering of some courses: Elaboration of Academic Articles; III quarter of 2013 and Elaboration of Academic Essays and Organization and Design of Online Courses; from I quarter of 2014, these training processes were developed in the virtual platform Moodle. These courses incorporate the subject of qualitative evaluation and formative evaluation both in theory and in practice, as is evidenced in the course evaluation and in the role that assumes the facilitator (trainer).
\end{abstract}

Keywords: qualitative evaluation, formative evaluation, authentic evaluation, online courses, distance education. 


\section{Introducción}

El Centro de Capacitación en Educación a Distancia (CECED) es un programa adjunto a la Vicerrectoría Académica. Su misión es ofrecer diversas actividades que potencien las habilidades pedagógicas y tecnológicas de los y las profesionales de la institución, especialmente de aquellas personas que participan en los procesos docentes ofrecidos por la Universidad Estatal a Distancia (UNED); de ahí que los proyectos participativos de capacitación son espacios para reflexionar críticamente sobre sus experiencias académicas, de cara a los avances en el campo de la didáctica y de las metodologías de la enseñanza a distancia (CECED, 2013).

Aunque dicho centro fue creado desde hace catorce años, no es sino a partir de 2004 que se reflexionó sobre su quehacer para comprometerse todavía más con el modelo pedagógico de la UNED, por lo que desde hace siete años se intensificó su propuesta de capacitación, pues mediante el uso de las plataformas virtuales se encontraron estrategias metodológicas más acordes con la modalidad a distancia y propias del contexto UNED. Así pues, para el 2007 el CECED replanteó la temática y la dinámica de cursos básicos que les ofrece a los/as funcionarios/as de la universidad, entre ellos Pedagogía Universitaria para la Educación a Distancia, curso que duraba más de dos meses y recurría a encuentros presenciales para desarrollar la mayoría de sus dinámicas. Además, toda la evaluación era responsabilidad de la persona facilitadora (capacitador) y las actividades obligatorias tenían un carácter meramente teórico.

Paralelamente, en ese mismo año, también se empezó a desarrollar la primera versión del curso Enseñar y Aprender en Entornos Virtuales, el cual se ofrecía en una modalidad híbrida (presencial y virtual). En ese momento la metodología del curso se organizó por módulos separados y las personas decidían en cuál de todos participar, consecuentemente, todas las actividades de los módulos eran 
independientes y no tenían relación entre sí; por lo tanto, la propuesta evaluativa no era tan integral e integradora, fundamento teórico indicado en el modelo pedagógico de la UNED. Asimismo, toda la evaluación era responsabilidad de la persona facilitadora (capacitador) y las actividades obligatorias tenían carácter teórico, de manera que lo práctico era muy limitado a lo largo de cada curso.

En el 2009 se empezó a trabajar el curso Evaluación de los Aprendizajes para los Entornos Virtuales de manera híbrida. En su programa se indicaba textualmente:

El propósito principal de este proceso de capacitación es que el participante comprenda, aplique y diseñe estrategias de evaluación que potencien los procesos de aprendizaje realizados por el estudiante en la UNED. De esta manera, el futuro profesional construirá y reconstruirá conocimientos, destrezas, habilidades y valores que le serán de gran utilidad para su carrera y su desempeño futuro a nivel laboral (CECED, 2009, p.1)

Tal como se evidencia en la cita anterior, desde entonces el CECED ha buscado estrategias de capacitación que potencien propuestas evaluativas que respondan al modelo pedagógico de la institución y que, además, sean acordes con las necesidades del contexto social y laboral de quienes participan de ella. Por estas razones las siguientes versiones de este curso y todos las demás ofertas del centro se enmarcan dentro de un enfoque de evaluación auténtica.

En consecuencia, desde el 2013 se empezó a trabajar el curso Organización y Diseño de Cursos en Línea (OYD) de acuerdo a una propuesta evaluativa más enfocada en los procesos y menos en los resultados, lo que a su vez propicia un aprendizaje más centrado en la realimentación y menos en la calificación como producto de la medición. Además, se propone que el curso promueva la evaluación como responsabilidad compartida, de manera que no prevalezca la tarea evaluadora de la persona facilitadora (capacitador). De esta forma se diseña la primera versión de este curso con una evaluación completamente cualitativa 
que establece una propuesta evaluativa para favorecer la calidad del proceso y el desempeño de las personas participantes.

Los resultados y las buenas experiencias de las primeras ofertas del curso OYD llevaron al CECED a reflexionar sobre la importancia y necesidad de implementar este tipo de estrategias evaluativas en otros cursos de capacitación, de forma que se continuó con el rediseño de Pedagogía Universitaria para la Educación a Distancia (PED), el cual, al igual que OYD, es de participación obligatoria para los/as funcionarios/as académicos/as.

El curso PED es el punto de referencia sobre qué, cómo, por qué y para qué es el modelo pedagógico de la UNED. Tal modelo da los lineamientos orientadores sobre cómo se gestiona y se desarrolla el quehacer docente enfocado en la centralidad del estudiante. Dicha centralidad también debe evidenciarse en la manera en que se realizan las prácticas evaluativas de los cursos y las asignaturas que se ofertan cada cuatrimestre en la universidad.

En este sentido, esta ponencia pretende compartir la percepción de algunas de las personas participantes que completaron exitosamente procesos de capacitación que incorporaron una propuesta de evaluación formativa y cualitativa. Para tal efecto se seleccionaron participantes de los cursos Elaboración de Artículos Académicos, del III cuatrimestre de 2013, Elaboración de Ensayos Académicos y Organización y Diseño de Cursos en Línea, del I cuatrimestre de 2014, desarrollados en la plataforma Moodle.

Estos aportes brindados por las personas participantes le permitirán al CECED tomar nuevas y mejores decisiones respecto al tema de la evaluación de los aprendizajes, pues todas las capacitaciones antes expuestas incorporan este tema tanto en lo teórico como en lo práctico, de manera que repercute no solo en el 
diseño propio de la estrategia evaluativa, sino en el papel que asume quien facilita la capacitación, por lo tanto, en las mismas personas participantes.

\section{Concepciones teóricas}

Tal como se indica en el Modelo Pedagógico de la Universidad Estatal a Distancia (2004), la evaluación de los aprendizajes no debe concebirse como:

- Un proceso aislado del curso o de la asignatura

- Cuantificación de respuestas correctas e incorrectas por parte del estudiante

- Una tarea exclusiva del equipo docente

- Un proceso en el que solo hay medición

- Sinónimo de aplicación de pruebas escritas

- Una manera de reforzar la memorización de contenidos

Por el contrario, en este documento se indica que mediante la evaluación de los aprendizajes se debe:

- Involucrar dentro del proceso de aprendizaje los conocimientos previos del estudiante

- Potenciar en los estudiantes la capacidad de aprender a aprender

- Favorecer la participación del estudiante de manera activa, consciente, voluntaria y reflexiva

- Promover el logro de los objetivos de aprendizaje de manera autónoma y autorregulada

- Proponer estrategias de aprendizaje relacionadas con el contexto en el que el estudiante se va a desempeñar como profesional

- Favorecer procesos de autoevaluación y coevaluación 
En ese sentido, cabe aclarar que por evaluación formativa se entiende:

El tipo de evaluación que se lleva a cabo durante todo el proceso de aprendizaje, la cual posee una función reguladora del proceso de enseñanza-aprendizaje a fin de posibilitar que los medios de formación respondan a las características del estudiantado. Tiende más a identificar cuáles son las deficiencias del aprendizaje que a determinar los resultados alcanzados con este aprendizaje (Jorba y Casellas, 1997, citado por PACE, 2013, p. 41).

Esto implica que la evaluación debe ser constante, pero sobre todo oportuna, pues es a partir de ella que la persona participante puede reflexionar sobre su proceso y progreso en el aprendizaje. Sin embargo, si se visualiza esta evaluación desde un enfoque cuantitativo se puede caer en el error de que lo que se requiere en todos los cursos o asignaturas es recargar las estrategias de aprendizaje-evaluación con actividades y herramientas de comunicación e interacción. En otras palabras, desde un punto de vista tradicionalista la propuesta evaluativa puede contener muchas actividades obligatorias para las cuales la persona participante obtiene frecuentes notas o porcentajes por su elaboración o presentación.

En ese sentido, cabe aclarar que evaluación cuantitativa y cualitativa no son sinónimos, pero tampoco son conceptos excluyentes. En la universidad, así como en la mayoría de centros de educación, las ofertas académicas han promovido más el enfoque cuantitativo que el cualitativo. Sobre tal aspecto Quesada indicó que:

En la primera se cuida la objetividad y el rigor. La segunda usa métodos más informales, aunque sistemáticos, para abundar en la información. La cuantitativa ha sido práctica común en la enseñanza tradicional, la cualitativa se ha usado poco porque su surgimiento es relativamente reciente. En la educación a distancia se incluyen ambas (García, 2001, citado por Quesada, s. f., p. 12).

Al respecto Luis Elías (s.f.) acotó que tanto el concepto de evaluación formativa como el de evaluación cualitativa están altamente relacionados con el concepto de evaluación auténtica pues este implica que la evaluación debe ser parte integral y 
natural del proceso de aprendizaje. Esto implica que se debe recurrir a diversos procedimientos y técnicas para evaluar los objetivos de aprendizaje alcanzados por el estudiante, tanto en estrategias de aprendizajes individuales como colectivas. Además, parte de la base de que la permanente integración de aprendizaje y evaluación por parte del propio estudiante y de sus pares, constituye un requisito indispensable del proceso de construcción y comunicación del significado. Así pues, dijo:

De acuerdo a esto, la evaluación contribuye a regular el proceso de aprendizaje; es decir, permite comprenderlo, retroalimentarlo y mejorarlo en sus distintas dimensiones y, en consecuencia, ofrece al profesor y al equipo docente la oportunidad de visualizar y reflexionar sobre el impacto de sus propias prácticas educativas, todo lo cual redundará, especialmente, en el mejoramiento de la calidad de los aprendizajes construidos por los estudiantes (p. 1).

De ahí que para implementar todos los elementos antes señalados el CECED llevó a cabo un proceso de reflexión sobre sus prácticas evaluativas, ya que, aun cuando se llevan a cabo a través de diferentes herramientas que ofrecen las plataformas virtuales, no es suficiente para promover la centralidad del estudiantado (en este caso, el funcionario académico que participa en tales capacitaciones). Al realizar un análisis de todas las propuestas evaluativas de los cursos del CECED se puede evidenciar lo siguiente:

- Todas las actividades obligatorias se evaluaban cuantitativamente.

- La mayoría de estas actividades son evaluadas por quien facilita la capacitación (capacitador).

- Por los plazos de cada actividad la persona participante no tenía oportunidad de retomar los aspectos brindados en la realimentación.

- Al no existir periodos para retomar la realimentación, los procesos y productos de aprendizaje no eran retomados ni mejorados. 
- Un gran porcentaje de dudas relacionadas con la evaluación de los aprendizajes, terminaban siendo provocadas por la nota o el porcentaje otorgado por la persona evaluadora.

Por lo anterior, el CECED, en busca de la innovación y la mejora continua, inició con un proceso de actualización del curso OYD y posteriormente con el de PED, con el fin de potenciar el aprendizaje y la estrategia de evaluación mediante procesos de evaluación cualitativos y formativos. Para ello creó instrumentos apropiados que permitan la realimentación pertinente y oportuna durante todas las actividades de dicha capacitación, de forma que las personas participantes puedan aprender de sus aciertos, errores, virtudes y dificultades de manera progresiva durante cada etapa del curso.

\section{Descripción de la experiencia de capacitación}

En primer lugar, se presentan dos cursos que, aunque no poseen una propuesta evaluativa $100 \%$ cualitativa, fueron los primeros diseñados en el CECED que centraron la estrategia evaluativa en lo formativo (en el proceso), más que en lo sumativo (en los resultados). De ahí que la realimentación de la persona facilitadora es fundamental para que quienes cursan la capacitación mejoren las sucesivas entregas de los productos solicitados:

\section{Elaboración de ensayos académicos}

Tiene como propósito promover la construcción de textos críticos y reflexivos en los equipos docentes de la UNED. Dicho curso es completamente virtual y está conformado por cuatro unidades. Durante todas las semanas los participantes van elaborando de manera progresiva un ensayo, para lo cual deben presentar evidencias del proceso de construcción, con el fin de ser realimentados por la persona facilitadora $y$, a partir de esa realimentación, seguir construyendo el 
ensayo hasta obtener una versión final. Este curso posee evaluación cuantitativa, mas no deja de lado la evaluación formativa y cualitativa.

\section{Elaboración de artículos académicos}

Tiene como propósito comprender el proceso para la elaboración y publicación de artículos académicos, con el fin de potenciar la práctica investigativa dentro de la UNED. Este curso es híbrido y casi todo se lleva a cabo a través de la plataforma virtual, pues solo tiene una sesión presencial obligatoria.

El curso está conformado por cuatro unidades. De igual forma durante todas las semanas las personas participantes van elaborando de manera progresiva un artículo académico que surgirá como resultado de una investigación previa. Para eso deben presentar evidencias de su proceso de construcción, con el fin de ser realimentadas por la persona facilitadora, y a partir de esos insumos seguir construyendo el artículo final. Al igual que el curso anterior, este también posee evaluación cuantitativa, pero tampoco deja de lado la evaluación formativa y cualitativa.

Estas dos experiencias de capacitación le evidenciaron al CECED la importancia y la necesidad de buscar una propuesta evaluativa más integral e integradora, pues fue indudable que la manera en la que se evaluaban los productos (ensayos y artículos) repercutía sobre su calidad y sobre la motivación de quienes participaban en los cursos, tal como se reflejó en la evaluación que realizaron las personas participantes al finalizar tales cursos. Ellas indicaban que fue muy significativo recibir realimentación constante para mejorar las siguientes entregas, pues les permitía avanzar en el trabajo de manera más segura (CECED, 2013). 
A raíz de esto, en segundo lugar, se mencionan los dos cursos que actualmente presentan una propuesta evaluativa 100\% cualitativa, lo cual implica que durante el curso no hay entrega de notas por las actividades realizadas:

\section{Organización y Diseño de Cursos en Línea (OYD)}

Tiene el propósito de desarrollar la programación de asignaturas/cursos en línea de grado, posgrado y extensión, según los criterios institucionales, con el fin de potenciar la calidad de la oferta académica de la UNED. La propuesta evaluativa original del curso indicaba lo siguiente:

\section{Tabla 1. Propuesta evaluativa original de OYD}

\begin{tabular}{ll}
\hline Actividades obligatorias & Porcentaje \\
\hline Tarea 1: Diseño de la matriz de programación & $10 \%$ \\
Foro: Gestión de la Virtualización & $10 \%$ \\
Tarea 2: Diseño de la matriz de programación (segunda parte) & $10 \%$ \\
Avance del espacio de simulación (diseño del aula virtual) & $10 \%$ \\
Chat: Aspectos Específicos sobre el Diseño del Aula Virtual & $10 \%$ \\
Tarea 3: Diseño de rutas de aprendizaje y consignas & $15 \%$ \\
Finalización del espacio de simulación (diseño del aula virtual) & $25 \%$ \\
Autoevaluación de cada unidad & $10 \%$ \\
\hline
\end{tabular}

Fuente: CECED, 2012. Programa del curso OYD

Al usar una propuesta evaluativa como la del ejemplo anterior, muchas de las preguntas y observaciones realizadas por parte de algunas personas participantes se centraban en el puntaje obtenido y en cómo hacer para recuperar los puntos perdidos. Además, era común que las personas facilitadoras del CECED enviaran recordatorios como: "Favor revisar la nota de la actividad, recordar que la nota 
mínima para aprobar el curso es de $80 \%$. Tienen 3 días para apelar la nota o hacer las consultas respectivas sobre las observaciones recibidas en la actividad".

No obstante, desde el II cuatrimestre de 2013 se empezó a utilizar una propuesta evaluativa completamente cualitativa, para lo cual se diseñaron instrumentos de evaluación que favorecen la autorregulación y la evaluación formativa por parte de la persona facilitadora (capacitador). Así pues, en la actualidad el curso es completamente virtual y está organizado en cuatro unidades.

En cada una de estas unidades las personas docentes que participan de la capacitación van construyendo una propuesta de virtualización para un curso o asignatura que se ofrece en la UNED (cátedras, posgrados, extensión). Esta propuesta se elabora en la matriz de programación y, con base en ella, se diseña el aula virtual. Esto implica que todo aquello que aprende el participante del curso deberá aplicarlo en su contexto docente real.

\section{Pedagogía universitaria para la educación a distancia}

Tiene el propósito de promover la calidad del proceso educativo a partir de la comprensión del modelo pedagógico en la educación a distancia de la UNED. De la misma forma que el curso anterior, esta capacitación presentaba una propuesta como la que se muestra a continuación: 
Tabla 2. Propuesta evaluativa original de PED

\begin{tabular}{ll}
\hline Actividades obligatorias & Porcentaje \\
\hline Diario reflexivo unidad 1 & $10 \%$ \\
Foro unidad 2 & $15 \%$ \\
Análisis sobre el diseño curricular y las orientaciones del curso unidad 3 & $15 \%$ \\
Foro unidad 4 & $15 \%$ \\
Diario reflexivo unidad 5 & $10 \%$ \\
Diagnóstico y análisis sobre los estudiantes de la UNED unidad 6 & $20 \%$ \\
Autoevaluación de cada unidad & $15 \%$ \\
\hline
\end{tabular}

Fuente: CECED, 2011. Programa del curso PED

Como se observa, al igual que en el curso anterior, durante esta capacitación se reforzaba la importancia de la calificación como producto del proceso de medición. Una de las grandes debilidades de esta propuesta evaluativa era que las actividades de cada unidad no tenían mayor relación y congruencia entre sí, sino que cada unidad se diseñaba de manera aislada y no facilitaba la integración de los aprendizajes.

Actualmente, este curso es completamente virtual y está organizado en cuatro unidades. Para el I cuatrimestre de 2014 se empezó a implementar la versión de curso en la que la propuesta evaluativa es completamente cualitativa y se reforzó el carácter formativo.

A partir de estos cambios y mejoras significativas en las propuestas evaluativas, el CECED requiere conocer la percepción de los participantes de estos cursos, en los que el carácter formativo y cualitativo es ahora más evidente y fundamental, no solo para aprobar el curso, sino como una estrategia que promueve aprendizajes más significativos, profundos y contextualizados. 
De esta forma, con el fin de conocer la percepción de algunas de las personas participantes en cursos de capacitación del CECED (sobre qué es y cómo poner en práctica estrategias de evaluación cualitativa y formativa en las ofertas académicas de la UNED), se encuestó a quienes participaron de la última oferta de los cursos Elaboración de Artículos Académicos, del III cuatrimestre de 2013, Elaboración de Ensayos Académicos y Organización y Diseño de Cursos en Línea, del I cuatrimestre de 2014.

Así pues, al preguntarles a las personas encuestadas (tutores y tutoras, encargados y encargadas de cátedra, así como extensionistas) sobre cómo conciben la evaluación de los aprendizajes, las ideas en su mayoría se refieren a lo siguiente:

- "Un medio para medir niveles de aprendizaje"

- "El seguimiento cualitativo y cuantitativo del proceso de aprendizaje"

- "Medición, ya sea formativa o sumativa o diagnóstica"

Tales ideas evidencian que, conceptual y tradicionalmente, se ha asociado la evaluación con términos relacionados con la medición y la cuantificación. Esto es lo que puede percibirse en muchos cursos o asignaturas que se ofertan en la UNED. En ese sentido, cabe resaltar que la capacitación no puede ni debe limitarse a este tipo de prácticas evaluativas, pues estaría en contra de nuestro modelo pedagógico.

Asimismo, las personas encuestadas expresaron que el proceso evaluativo puede generar diversas emociones y sentimientos, generalmente contrapuestos: 
Tabla 3. Sentimientos y emociones generados por la evaluación

\begin{tabular}{ll}
\hline Positivos & Negativos \\
\hline Satisfacción & Frustración \\
Compromiso & Cansancio \\
Nuevos retos & Temor por la incertidumbre \\
Expectativas por hacer mejor las & Contradicción \\
cosas & \\
Alegría & Conflicto \\
\hline
\end{tabular}

Fuente: elaboración propia

De acuerdo con una de las personas participantes de la encuesta, estos sentimientos y emociones son generados, principalmente, cuando la evaluación es tradicional, pues si no está bien fundamentada y desarrollada puede provocar desde ansiedad hasta temor a fallar. Todo ello porque generalmente dentro de un proceso de enseñanza-aprendizaje prima la calificación, el resultado final de un producto solicitado.

Debido a ello fue común encontrar que las personas encuestadas creían más pertinente realizar una evaluación que incluyera tanto aspectos cualitativos como cuantitativos, y no solo estos últimos. Lo anterior se demuestra en el siguiente gráfico: 
Gráfico 1. Información más útil para el curso. Según su percepción ¿cuál información es más útil para el curso: las calificaciones que se obtienen de las actividades, la realimentación recibida durante las actividades, 0 ambas? ¿Por qué?

\section{Información más útil para el curso}

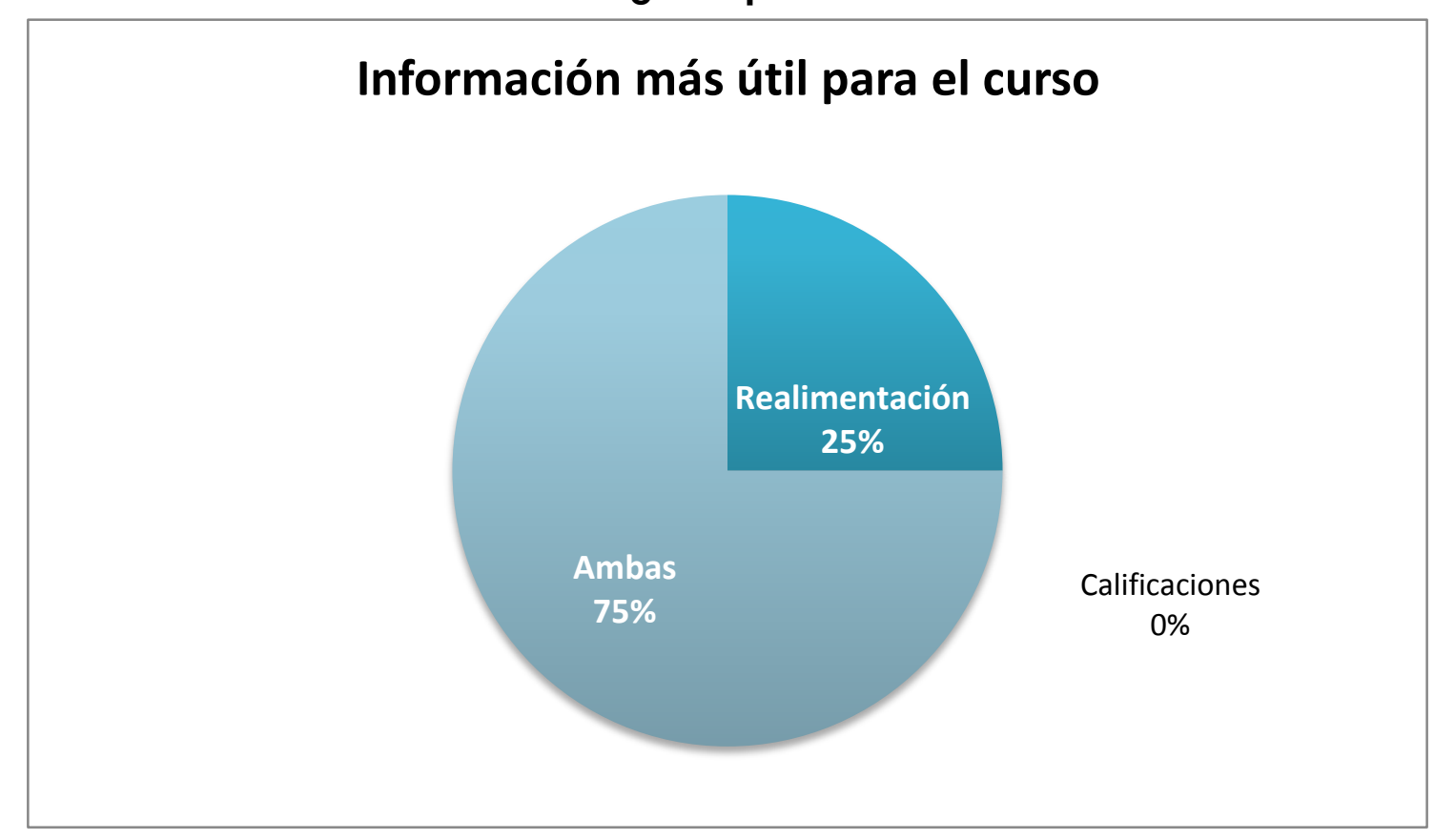

Fuente: elaboración propia

De acuerdo con los datos presentados y los insumos brindados por las personas encuestadas, la evaluación que incluye tanto una realimentación oportuna como una calificación, que permita determinar el nivel de avance del estudiantado en los diversos cursos de los cuales forma parte, es fundamental para su aprendizaje. Por ello, se descarta que el nivel de aprendizaje y aprovechamiento durante la capacitación hubiese sido mayor de ser evaluado en su totalidad solo con porcentajes y notas.

En este sentido, afirmaron que "la nota no indica lo que realmente se aprende, lo más importante es el conocimiento, no un porcentaje", o bien que la nota "no implica que haya aprendido, ya que es necesario contar con valoraciones formativas para ver en qué se está fallando". Esto, a su vez, reafirma la 
importancia de la realimentación dentro del proceso de capacitación, en este caso, pues consideraron que es un "apoyo y guía" para el estudiantado. Por ello, a la pregunta "¿Cree que su nivel de aprendizaje y aprovechamiento durante el curso hubiera sido mayor si se hubiera evaluado sin ningún tipo de realimentación durante cada unidad que conforma el curso? ¿Por qué?", la totalidad de los encuestados contestó de manera negativa. Algunos comentarios al respecto se muestran a continuación:

- "No, en estos procesos siento que la realimentación es importante, porque se convierte en un complemento esencial del curso".

- "No para nada, por el contrario, la realimentación es importante en todo proceso de enseñanza-aprendizaje y considero que es un derecho del participante recibir algo más que su calificación".

Finalmente, todos coincidieron en que la realimentación constante durante el desarrollo de las unidades de todo el curso fue determinante para potenciar el aprendizaje y desempeño, ya que la consideran "pieza clave del proceso de aprendizaje", "permite un panorama más amplio de los aprendizajes" y "permite ver avances durante, en el momento y después".

Otras de las preguntas de la encuesta se referían a la forma de evaluación (heteroevaluación, coevaluación o autoevaluación) mediante la que prefieren ser evaluados los participantes en las capacitaciones del CECED, así como al momento en que consideran más pertinente que se lleve a cabo este proceso. Los resultados se muestran en los siguientes gráficos: 
Gráfico 2. Formas de evaluación. ¿Cómo prefiere ser evaluado: por el facilitador, por un compañero o por usted mismo? ¿Por qué?

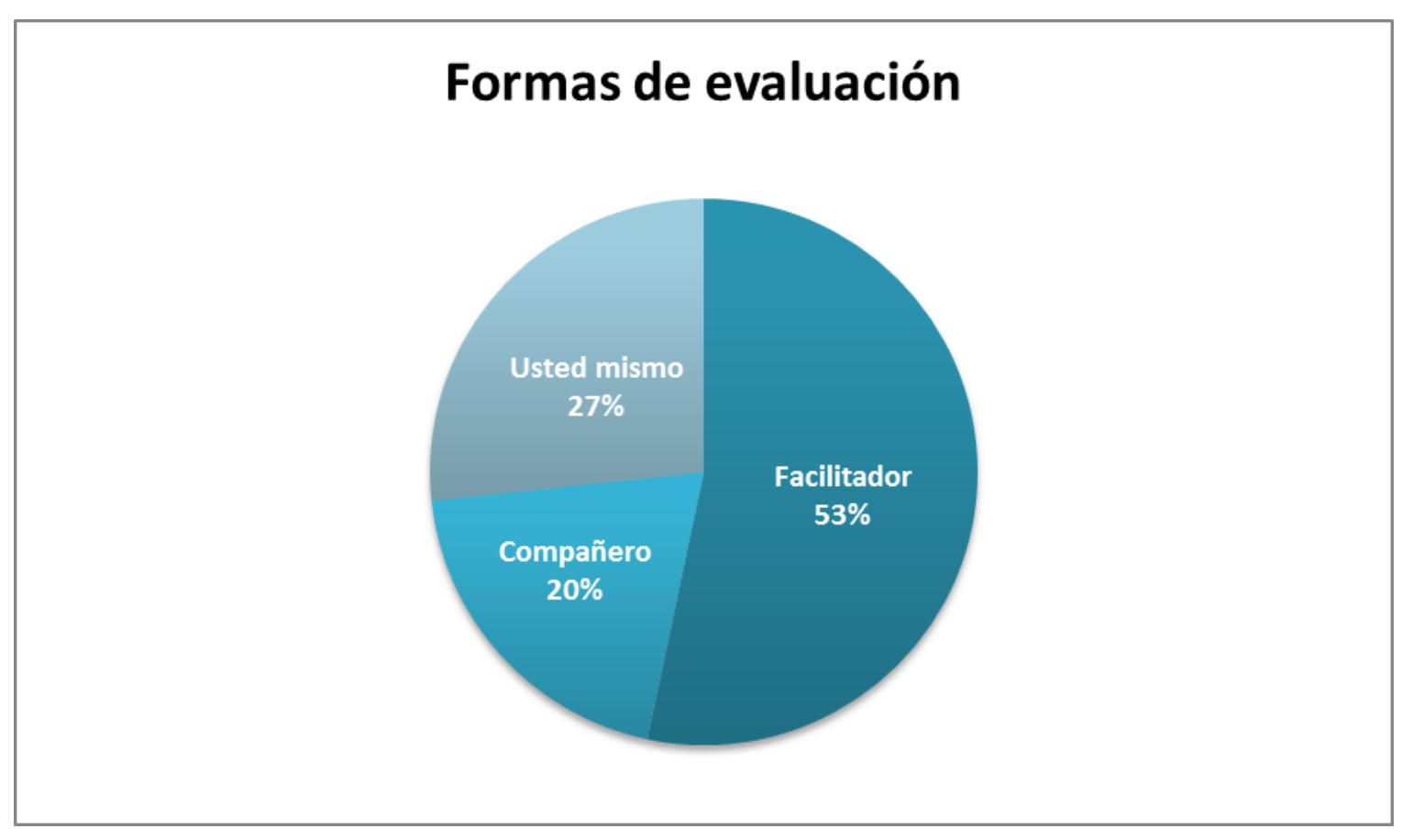

Fuente: elaboración propia

Al respecto, las personas encuestadas arrojaron respuestas como las que se exponen a continuación:

- "Por el facilitador, porque puede ser más objetivo, puede entender el avance en el aprendizaje y tiene experiencia en evaluación".

- "Por el facilitador y un compañero, porque la evaluación es más objetiva".

- "Me parece que la retroalimentación es importante para mejorar, por lo cual es importante ser evaluado por el facilitador y los compañeros".

- "Por el facilitador y por mí misma, ya que considero que en algunos casos los compañeros no pertenecen a la misma cátedra y tienen otra forma de ver la evaluación". 
Como se observó, las respuestas predominantes sobre quién debe llevar a cabo la evaluación indican que debe ser una labor tanto de la persona facilitadora como de los mismos participantes. Lo anterior implica que la mayoría está consciente de que la realimentación se enriquece si también se toma en cuenta el punto de vista de quienes participan en el proceso de enseñanza-aprendizaje, pues eso permite ampliar el enfoque de los comentarios, las observaciones y las recomendaciones.

Además, esta percepción se complementa con la pregunta sobre cuándo debería llevarse a cabo la evaluación. La mayoría tiene claro que este debería ser un proceso continuo, por lo que si solo se realiza la evaluación al final del curso, del módulo o de la unidad se pierden muchos momentos valiosos para mejorar o redireccionar el rumbo del proceso de aprendizaje.

Lo anterior implica que la evaluación no solo se debe hacer en determinados momentos para otorgar un puntaje o porcentaje como resultado de un proceso de medición, sino que, más allá de la calificación, permite que la persona que aprende sea consciente de su propio aprendizaje, reflexione sobre si requiere hacer ajustes oportunos y pertinentes para lograr un mejor desempeño durante la actividad, incluso más allá del curso. En el gráfico que se presenta a continuación se refleja lo expuesto: 
Gráfico 3. Momento de la evaluación. ¿Cuándo prefiere ser evaluado: al inicio, durante $o$ al final de un tema $o$ unidad, durante $o$ al final de una actividad? ¿Por qué?

\section{Momento de la evaluación}

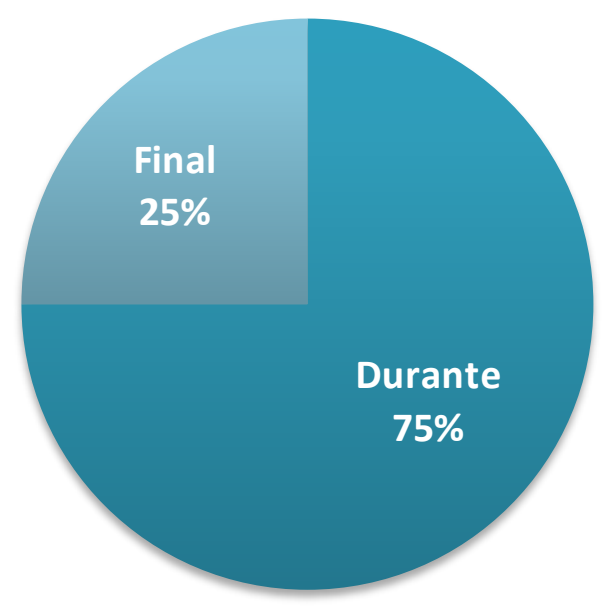

Fuente: elaboración propia

También se cuestionó sobre la importancia de la realimentación. De esta manera, a la pregunta ¿Cree usted que la realimentación constante durante las respectivas unidades fue determinante para potenciar su nivel de aprendizaje y desempeño durante el curso? ¿Por qué? Todas las personas participantes de la encuesta concuerdan en que la realimentación constante durante los cursos de capacitación definitivamente potenció su nivel de aprendizaje y desempeño durante el curso.

Algunas justificaciones indican lo siguiente:

- "[La realimentación es una] pieza clave del proceso de aprendizaje".

- "[La realimentación sirvió de] guía sobre lo que se quería de mí como estudiante y cómo llegar a mi objetivo". 
- "[Mediante la realimentación] se iban mejorando aspectos que podían ser influyentes en el proceso de aprendizaje significativo".

- "[La realimentación] permite tener un panorama más amplio de los aprendizajes".

- "[La realimentación es una] radiografía de la situación".

- "[La realimentación] permitió saber que estaba logrando los objetivos. Además de la guía para mejorar o cambiar lo que era necesario".

Tales ideas reflejan que la evaluación, más allá de lo cuantitativo, requiere de lo cualitativo y lo formativo para que la persona encuentre significado y motivación durante el proceso de aprendizaje. Además, dichas respuestas reflejan que este tipo de evaluación permite el mejoramiento de los aspectos débiles y la reafirmación de los aspectos fuertes en ese proceso de construcción y reconstrucción del conocimiento, no solo resalta lo negativo o los errores de una actividad o un producto solicitado durante una unidad o un módulo de un curso.

Finalmente, al cuestionar si consideraban que en los cursos o las asignaturas que se imparten en la UNED podrían implementarse propuestas de evaluación formativa y cualitativa, la totalidad de las personas encuestadas respondió de manera afirmativa, pero que para implementarla se deberían tomar en cuenta algunas ideas o recomendaciones. Desde lo cualitativo cabe resaltar algunas ideas expresadas ante la siguiente pregunta: “¿Cree que dentro de los cursos 0 las asignaturas que usted imparte en la UNED podría implementar más estrategias de aprendizaje que incorporen procesos de evaluación cualitativa (con realimentación pertinente)? ¿Cómo?”.

- "Promover el uso de plataformas de tecnologías de comunicación más ágiles que no despersonalicen la relación con el estudiante y que permitan una cercanía más inmediata a sus dudas".

- "Utilizar los portafolios de procesos, son estrategias o herramientas que permiten evaluar durante todo el proceso". 
- "Utilizar el chat y los foros, son indispensables".

- "Promover cualquier actividad que permita mostrar evidencias de comprensión de los alumnos".

- "Permitir que el estudiante sea consciente del avance o retroceso en su aprendizaje, su nivel de compromiso con el aprendizaje".

Desde lo formativo, las personas encuestadas se comprometieron a lo siguiente a partir de esta pregunta: "¿Cree que dentro de los cursos o las asignaturas que usted imparte en la UNED podría implementar más estrategias de aprendizaje que incorporen procesos de evaluación formativa (durante el proceso de aprendizaje de manera oportuna)? ¿Cómo?”.

- "Proponérmelo como meta y cumplirla en el próximo curso".

- "Variar la estrategia de evaluación y promover en el estudiante un cambio en la forma de ver sus esfuerzos, para que deje de verse a sí mismo reflejado en una calificación cuantitativa".

- "Incorporarlo a las giras de campo y talleres".

- "Ser creativos, la idea no es usar una estrategia porque existe, sino por el resultado que pueda dar".

- "Permitir el autoaprendizaje y la conciencia del estudiante hacia su propio aprendizaje".

Estas respuestas, así como las dadas en el resto de la encuesta, evidencian la necesidad y el anuncio de un cambio en la evaluación de los aprendizajes dentro de la universidad, así como una experiencia satisfactoria del proceso de capacitación por parte de quienes han sido evaluados desde un enfoque cualitativo, integral e integrador, como el propuesto en los cursos en estudio. 


\section{Conclusiones}

A partir de las respuestas brindadas por las personas participantes de los cursos de capacitación en estudio es posible determinar que:

- La evaluación cuantitativa no debe ser el único enfoque de evaluación, pues no siempre refleja los aprendizajes reales de quienes forman parte de un proceso de enseñanza-aprendizaje.

- Cuando un curso o una asignatura es evaluado únicamente desde un enfoque cuantitativo se genera un ambiente de tensión, frustración e incluso temor, pues se sabe que lo principal es la suma de errores y aciertos que obtenga quien aprende y no las posibilidades de mejora que sus evaluaciones pueden arrojar.

- La evaluación cualitativa y formativa es fundamental para el logro de aprendizajes de calidad, significativos y duraderos, así como para lograr la motivación en el estudiantado.

- La evaluación debe ser un proceso continuo que permita redireccionar el rumbo del proceso de aprendizaje en caso de ser necesario, así como la reflexión y conciencia por parte de quien aprende sobre su propio aprendizaje.

- La realimentación es indispensable en todo proceso evaluativo, pues esta es complemento del aprendizaje del estudiantado. Funciona como apoyo y guía para quien aprende sobre qué aspectos puede mejorar, o bien cuáles aprendizajes están superados.

\section{Referencias bibliográficas}

Centro de Capacitación en Educación a Distancia (CEDED). (2009). Evaluación de los Aprendizajes para Entornos Virtuales. Programa de curso. Universidad Estatal a Distancia, Costa Rica.

Centro de Capacitación en Educación a Distancia (CEDED). (2011). Pedagogía Universitaria para la Educación a Distancia. Programa de curso. Universidad Estatal a Distancia, Costa Rica. 
Centro de Capacitación en Educación a Distancia (CEDED). (2012). Organización y Diseño de Cursos en Línea. Programa de curso. Universidad Estatal a Distancia, Costa Rica.

Centro de Capacitación en Educación a Distancia (CEDED). (2013). Informe de evaluaciones (manuscrito sin publicar). Universidad Estatal a Distancia, Costa Rica.

Luis, E. (s.f.). La evaluación auténtica de los aprendizajes. Recuperado de: http://www2.minedu.gob.pe/digesutp/formacioninicial/wpdescargas/bdigital/025_evaluacion_autentica.pdf

Programa de Apoyo Curricular y Evaluación de los Aprendizajes (PACE). (2013). Glosario de términos curriculares para la Universidad Estatal a Distancia. Recuperado de: http://www.uned.ac.cr/academica/index.php/pace/inicio

Quesada, R. (s.f.). Evaluación del aprendizaje en la educación a distancia «en línea». Facultad de Psicología y Dirección General de Evaluación Educativa. Universidad Nacional Autónoma de México. Recuperado de: http://www.um.es/ead/red/M6/quesada.pdf

Universidad Estatal a Distancia (2004). Modelo Pedagógico de la Universidad Estatal a Distancia. Aprobado por el Consejo Universitario, sesión № 1714 (artículo IV, inciso 3), del 9 de julio de 2004. San José, Costa Rica: EUNED. 